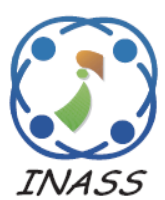

\title{
MIMO Radar Array Antenna with Transmit-Receive Subarrays
}

\author{
Syahfrizal Tahcfulloh ${ }^{1 *}$ \\ ${ }^{1}$ Department of Electrical Engineering, Universitas Borneo Tarakan, Tarakan, 77123, Indonesia \\ * Corresponding author’s Email: rizalubt@gmail.com
}

\begin{abstract}
Unlike the PMIMO radar, the transmit-receive (Tx-Rx) subarrays MIMO (TRSM) radar uses overlaping subarrays in Tx and Rx array so that it simultaneously combines the main advantage of the phased array radar (PA) i.e., high directional coherent gain, and the main advantage of the MIMO radar i.e., high waveform diversity gain. This paper has derived the radar performance formula such that Tx-Rx gain and SINR. The approach aims to overcome the beam shape loss, increase the transmit-receive gain, minimize the maximum peak sidelobe levels, narrow the half power beamwidth, increase directivity, and increase signal-to-noise-plus-interference ratio (SINR). This radar's performance is compared to the PMIMO radar in various methods such as equal subarrays, unequal subarrays, and optimum partitioning, the PA radar, and the MIMO radar. The numerical simulation and evaluation results show that the proposed radar has several advantages such as lowest the peak sidelobe level, narrow the half power beamwidth, and high directivity, so it is very robust against interference effects. When compared to the OPPM radar, as representative of the best-performing radars, this radar has an average performance improvement of MPSLL, directivity, and HPBW which are $21.5 \mathrm{~dB}, 2 \mathrm{~dB}$, and $0.45 \mathrm{deg}$, respectively.
\end{abstract}

Keywords: Array antenna, Coherent gain, MIMO radar, Phased array, Transmit-receive subarray.

\section{Introduction}

The phased array (PA) radar with its beam steering capability produces high directional coherent gain towards the target [1]. This directional coherent gain is useful for detecting weak targets and minimizing sidelobe levels in the direction of interference [2]. Unlike the PA radar, the multi-input multi-output (MIMO) radar has omnidirectional transmission by all transmit (Tx) elements, which are orthogonal to each other so as to produce high waveform diversity gain [3,4]. If these waveforms are perfectly orthogonal [5], they increase the channel capacity of the communication system [6] and provide efficient power allocation [7].

The MIMO radar equipped with signal processing can detect multiple targets $[8,9]$ with high resolution [10], high sensitivity [11], expand the detection range [12], and increase the parameter estimation [13]. However, the MIMO radar that is good in waveform diversity gain turns out poorly in the formation of directional coherent gain. This gain is very important for target detection and tracking [14]. This problem can be overcome by using the Phased-MIMO (PMIMO) radar approach, which exploits the main advantage of the PA radar i.e., directional coherent gain, and the main advantage of the MIMO radar i.e., waveform diversity gain simultaneously [15]. In addition, the PMIMO radar is still being studied to date because it has other advantages such as increasing the angular resolution, adding the identifiability parameters, expanding the aperture array, and increasing the target detection [16].

In principle, the PMIMO radar is the MIMO radar whose elements of the Tx array are overlapped subarrays with frequency variations to produce range resolution [17], radar ambiguity function settings [18], and optimization of Tx beampattern [19]. These subarrays are orthogonal to form directional beams such as PA with the number of elements of the subarray in the Tx array being equal [15-19] and unequal $[20,21]$. The purpose of using Tx subarray is to obtain high directional coherent gain as well as 


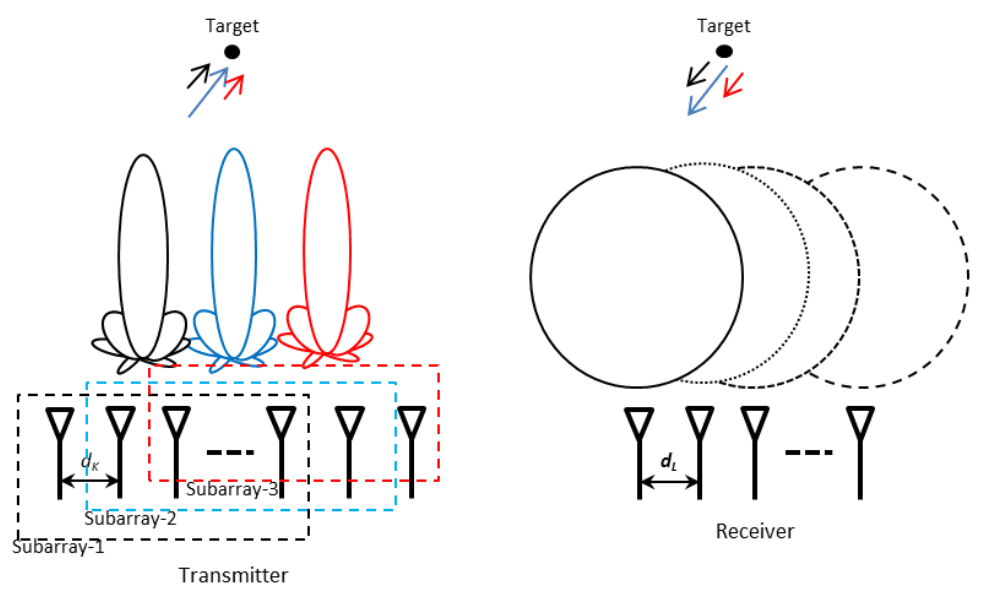

Figure. 1 Illustration of transmit-receive array for the PMIMO radar

to reduce the maximum peak sidelobe level (MPSLL) to be lower than the PA radar but on the other hand beam shape loss occurs on the mainbeam of the transmit-receive beampattern ( $\mathrm{T}-\mathrm{R}$ beampattern). MPSLL is the value of the sidelobe level in $\mathrm{dB}$ for the first sidelobe of mainlobe or mainbeam. This also shows that when MPSLL on PMIMO radar is low, directivity becomes large. In the study reported by [22], the optimum partitioning method has obtained the optimum number of subarrays that has the lowest MPSLL and highest directional gain compared to the previous method. Although the optimum number of subarrays has been obtained, the directivity gain obtained is not an optimum directivity. The optimum directivity produces half power beamwidth (HPBW), which focuses on the intended target and at the same time increases the ability to suppress interference. So that these conditions require the radar performance that has a high Tx-Rx gain as well as a high signalto-interference-plus-noise ratio (SINR).

This paper presents a Tx-Rx subarrays approach to the MIMO radar (TRSM) where both the Tx and $\mathrm{Rx}$ arrays are divided into overlapping subarrays. Each subarray in the Tx-Rx array has the same number of elements. The variation of waveform produced by the combination of the number of subarrays in the $\mathrm{Tx}-\mathrm{Rx}$ array compensates for the beam shape loss and the directivity compared to when using only subarrays on the Tx array as in the PMIMO radar. An optimum variation of the waveform occurs simultaneously increasing the directional coherent gain and the waveform diversity gain, so that it has a significant effect on increasing the radar performance such as low MPSLL, high directivity, narrow HPBW, and high SINR.

In this paper expand the discussion about the effect of the number of subarrays on the MPSLL value and beam shape loss from the study conducted by [15] so that the optimum subarray is found. The performance of the optimum number of subarrays is then compared with the radar performance investigated by [19] and [20]. The study by [19] states that the optimum number of subarrays from PMIMO radar is $K / 2-1$ where $K$ is the total number of antenna elements in the Tx array, whereas according to [20] that the radar performance of Tx-Rx gain and SINR has increased when using unequal subarray on $\mathrm{Tx}$ array or called Hybrid Phased MIMO radar with Unequal Subarrays (HPMR-US). Furthermore it was also proved that with the optimum partitioning PMIMO radar (OPPM) by [22] which produced a low MPSLL it did not provide optimum directivity. Optimum directivity determines the radar's HPBW and SINR. Overall this paper shows the comparison of the performance of the MPSLL, the T-R beampattern, directivity, HPBW, and SINR Output between the TRSM radar and other radars.

This paper presents the following matters. Section 2 reviews the subarray methods on the PMIMO radar and also provides an overview of the proposed radar i.e. the TRSM radar. Section 3 reviews and analyses Results and Discussion including drawings. The end of this paper is concluded in Section 4.

\section{Materials and methods}

\subsection{Review of subarray methods in phased-MIMO radar}

The PMIMO radar was introduced by [15]. The approach is carried out by dividing the Tx array into several subarrays which overlap with the number of elements of the antenna whose equal has been investigated by [15-19] and the number of elements of the antenna is unequal by [20,21]. The PMIMO's illustration with equal subarray is shown in Fig. 1 [25, 


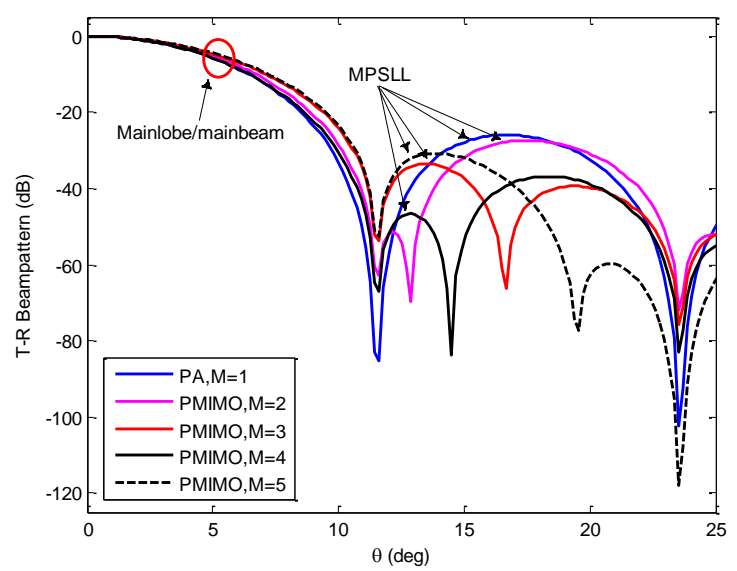

(a)

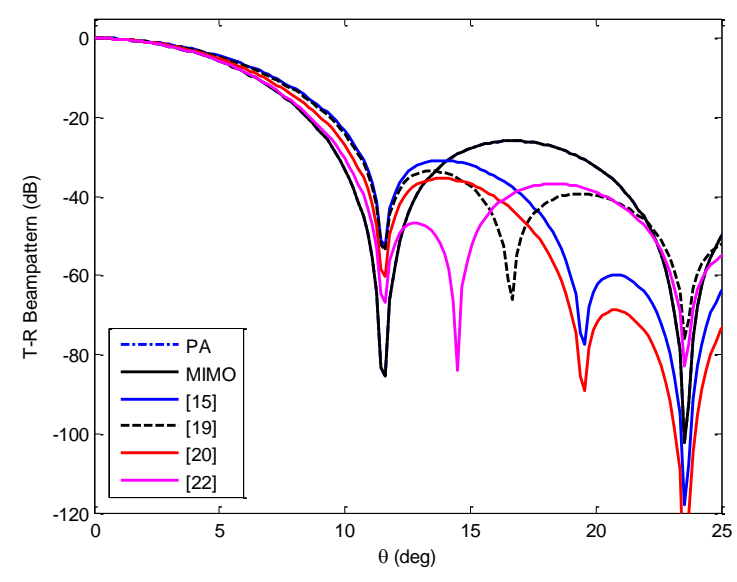

(b)

Figure. 2 T-R beampattern of the PMIMO radar with $K=L=10$ for: (a) variation of $M$ and (b) variety methods

modification of Fig. 1]. The optimum number of subarrays $(M)$ according to study by [19] is $K / 2-1$ whereas in study [22] that the optimum number of subarrays of PMIMO radar is $(K / 2)-[(K-1) / 12]$. In studies reported by [20] that the radar performance in the term of T-R gain and SINR has increased when unequal subarray is used in the Tx array. In summary, from the application of the three approaches, it is indicated that the selection of the right number of subarrays in the Tx array affects the MPSLL value which at the same time impacts the mainbeam directivity and the HPBW.

Fig. 2 (a) is a simulation of T-R beampattern from the PA and the PMIMO radar with varying number of subarrays starting from $M=1$ to $M=5$, the total number of antennas on Tx and Rx arrays is $K=L=$ 10 and the target at $\theta_{t}=0^{\circ}$. If it is desired that the MPSLL obtained is the lowest and the directivity obtained from mainbeam is narrow or no beam shape loss occurs, then there must be a mechanism to determine the optimum number of subarrays. The lowest MPSLL value obtained by the PMIMO radar is $M=2$ but it has a smaller directivity than the PA radar. For the PMIMO radar with $M=5$ or the number of subarrays which is half of $K$, the MPSLL value is apparently higher than the MPSLL value for PMIMO $(M=2)$. Also seen by varying the number of subarrays, the lowest MPSLL value is not always obtained in subarray $K / 2-1$ [19] or also in $(K / 2)-$ $[(K-1) / 12][22]$ or even for other number of subarray. Therefore we need an effort that can compromise the number of subarrays in order to obtain the performance of radar that has the lowest MPSLL and without sacrificing its directivity which should be better than its directivity of the PA radar. Low MPSLL, high directivity, and narrow HPBW are achieved so the consequence of high SINR is also obtained.
The subarray optimum effect on the radar performance for various radar methods such as the PA, the MIMO, the PMIMO radar [15], the PMIMO radar with the optimum number of subarrays $M_{o}=$ $K / 2$ - 1 [19], the PMIMO radar with unequal subarray or HPMR-US [20], and the OPPM radar, $M_{o}=(K / 2)$ $-[(K-1) / 12]$ [22] has been shown in Fig. 2 (b). With the OPPM method it is obtained that MPSLL is indeed the lowest but its directivity is still lower than PA radar. This is because the PMIMO radar type utilizes a simultaneous compromise between directional coherent gain and waveform diversity gain only on the Tx array while the Rx array does not have both of these gains. However, if the subarray method is also applied to Rx array, it has the potential to compensate for the lack of directivity or beam shape loss so as to produce high directivity while having a lower MPSLL. Numerical evaluations by [23] applying the use of subarray on Tx-Rx array have shown that the Tx-Rx gain and SINR obtained significantly increase compared to PMIMO radar performance. Thus the various subarray optimum methods on the PMIMO radars with subarray only on Tx array in principle only produce mainbeam whose directivity is lower than the PA radar.

In Fig. 3 (a) shows the effect of the number of subarrays on the MPSLL values of the PMIMO radar in study [15] with various of $K$. The number of subarrays determine the desired MPSLL or MPSLL can be adjusted through the determination of the number of appropriate subarrays. The trend of the MPSLL curve obtained is similar to the MPSLL curve reported by [22]. For Tx array with $K$ elements, there are some optimum number of subarrays $M$ which has the lowest MPSLL value following a certain formula as in the study by [19] and [22]. For example, for $K=\{49,50,51\}$ each has the lowest MPSSL value of $-34.41 \mathrm{~dB}$ for two subarrays i.e. for 


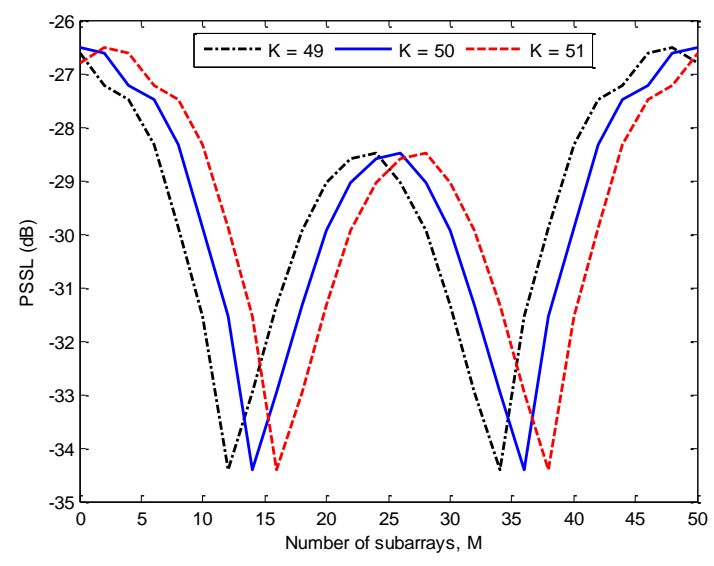

(a)

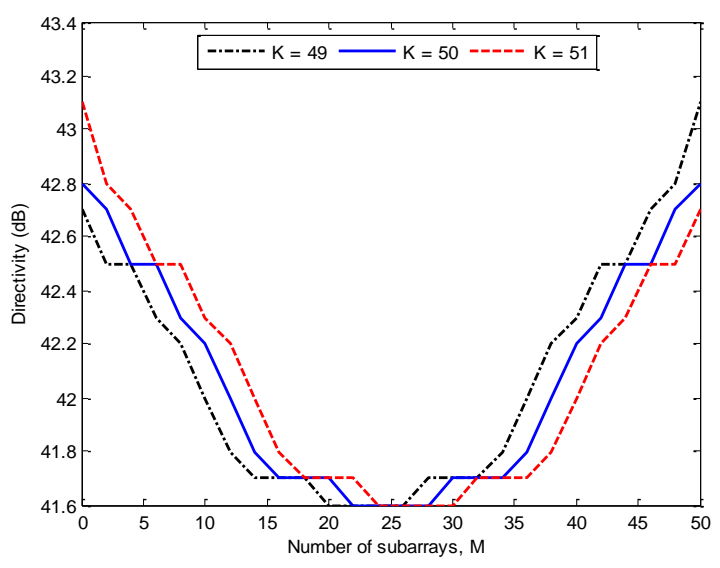

(b)

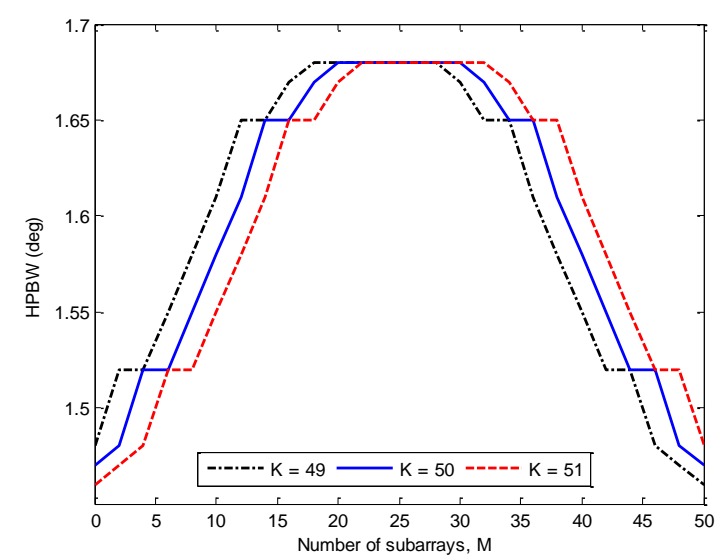

(c)

Figure. 3 The performance of the PMIMO radar against of $M$ at variation of $K$ for: (a) MPSLL, (b) directivity, and (c) HPBW

$K=49$ at $M=12$ and $M=34$, for $K=50$ at $M=14$ and $M=36$, and for $K=51$ at $M=16$ and $M=38$. For the number of subarrays at $M=K / 2$, that is $M=$ $\{24,25,26\}$ of the various $K$ has a lower MPSLL of $4.5 \mathrm{~dB}$ than MPSLL the optimum number of the subarrays. It also agrees with the results reported by [22].

For the directivity value of the PMIMO radar with various $K$ shown in Fig. 3 (b), it appears that when $M$ $=K / 2$ has the lowest directivity value i.e. $41.6 \mathrm{~dB}$. Whereas at $K=49$ with $M=12$ and $M=34, K=50$ with $M=14$ and $M=36$, and $K=51$ with $M=16$ and $M=38$ which have the lowest MPSLL it turns out that the directivity is $1 \mathrm{~dB}$ lower than directivity of the PA $\operatorname{radar}(M=1$ for $K=50)$ i.e. $42.8 \mathrm{~dB}$.

For HPBW values of the three variations of $K$, namely 49, 50, and 51, which are shown in Fig. 3 (c), it appears that for $K=50$ with $M=14$ and $M=36$ has a HPBW value i.e. $1.65^{\circ}$, while for $M=K / 2=25$ has a HPBW value i.e. $1.68^{\circ}$. As a comparison for the PA $\operatorname{radar}(M=1$ for $K=50)$ it turns out to have a HPBW value i.e. $1.47^{\circ}$. In summary, for optimum conditions of subarray with the lowest MPSLL has a relatively narrow HPBW and relatively large directivity. These conditions improve the radar performance in an effort to improve the ability of the target detection and suppress interference. HPBW in degree unit has a value which is inversely proportional to directivity. This means that high directivity radar has a narrow HPBW. It should be noted that a narrow HPBW or so-called pencil beam is very useful if desired radar works as the tracking radar. Based on the MPSLL, directivity, and HPBW values of a radar from an optimum number of subarrays, it is used as a basis for determining the optimum number of subarrays in the Tx-Rx subarrays method of the MIMO radar (TRSM).

In the PMIMO radar study by [15] it was assumed that the radar system with colocated antennas had $K$ antenna on the Tx array and $L$ antenna on the Rx array. Spaces between Tx and Rx antenna elements are $d_{K}$ and $d_{L}$, respectively. The transmitted signal is assumed to be a narrowband signal and its propagation is non-dispersive. The Tx array has been partitioned into $M$ subarray overlapping one another 

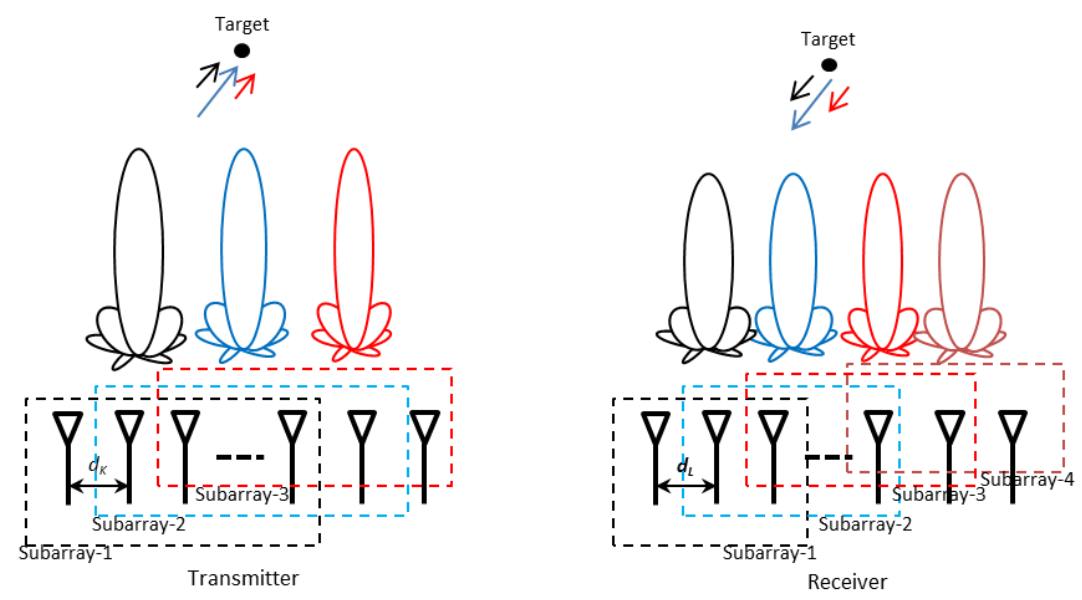

Figure. 4 Illustration of transmit-receive array for TRSM radar with $(M=3, N=4)$

as in Fig. 1. The number of antenna elements in each subarray is $K-M+1$ for the Tx array.

A beam is formed in each subarray in the direction of a certain target. Simultaneously each subarray on the Tx array emits a unique orthogonal waveform. The $m$-th subarray on the Tx array emits signals $\varphi_{m}(t)$ which are orthogonal to each other with other subarray signals. A beamforming weight vector is designed so as to maximize the coherent processing gain and waveform diversity gain on the subarray in the Tx array expressed by $M \times 1$ coherent vector transmit $\mathbf{c}(\theta)$ and vector transmit diversity $\mathbf{d}(\theta)$ as following [15].

$$
\begin{aligned}
& \mathbf{c}(\theta)=\left[\begin{array}{llll}
\mathbf{w}_{1}^{H} \mathbf{a}_{1}(\theta) & \mathbf{w}_{2}^{H} \mathbf{a}_{2}(\theta) & \cdots & \mathbf{w}_{M}^{H} \mathbf{a}_{M}(\theta)
\end{array}\right]^{T} \\
& \mathbf{d}(\theta)=\left[\begin{array}{llll}
e^{-j 2 \pi f \tau_{1}(\theta)} & e^{-j 2 \pi f \tau_{2}(\theta)} & \cdots & e^{-j 2 \pi f \tau_{M}(\theta)}
\end{array}\right]^{T}
\end{aligned}
$$

where $(\cdot)^{H}$ is a Hermitian transpose operation, $\mathbf{w}_{m}$ is a unit-norm complex weight vector with $K$ elements for the $m$-th subarray on the Tx array which has $K-M+$ 1 beamforming weights according to the active antenna element on the $m$-th subarray, so that the number of non-zero numbers in $\mathbf{w}_{m}$ is equal to $K-M$ +1 and the others are zero, the number $M-1, \mathbf{a}_{m}(\theta)$ is a transmit steering vector of size $K \times 1$ in the $m$ subarray, $\lambda$ is the carrier signal wavelength, $\tau_{m}(\theta)$ is the relative delay of the first antenna element of the $m$-th subarray to the first element of the first subarray, $\tau_{m}(\theta)=m d_{K} \sin (\theta) / c$ where $c$ is the speed of light.

Furthermore, from the coherent transmit vector $\mathbf{c}(\theta)$ and diversity transmit vector $\mathbf{d}(\theta)$ obtained in the $\mathrm{Rx}$ array, the $M L \times 1$ of $\mathrm{Tx}-\mathrm{Rx}$ steering vector is expressed by [15].

$$
\mathbf{u}_{\mathrm{PMIMO}}(\theta)=(\mathbf{c}(\theta) \circ \mathbf{d}(\theta)) \otimes \mathbf{b}(\theta)
$$

where $\odot$ represents the Hadamard product, $\otimes$ is the Kronecker multiplication operator, and the receive steering vector $\mathbf{b}_{n}(\theta)$ is similar to $\mathbf{a}_{m}(\theta)$. All vectors in $\mathbf{u}_{\text {PMIмо }}(\theta)$ namely $\mathbf{c}(\theta), \mathbf{d}(\theta)$, and $\mathbf{b}(\theta)$ are very dependent on the number of subarrays $M$ on the Tx array. For radar in study [15], the number of subarrays are $K / 2$, whereas in [19] that is $(K / 2)-1$. In HPMR-US [20], the number of subarrays are unequal and between subarray does not have a phase difference so that the diversity vector transmit component $\mathbf{d}(\theta)$ in (3) is $\mathbf{1}$, and the OPPM radar [22] has the number of subarrays expressed by $(K / 2)-[(K$ $-1) / 12]$.

\subsection{Antenna arrays of MIMO radar with transmit-receive subarrays}

The weakness of various PMIMO radar methods with the subarray technique only in the Tx array is the existence of beam shape loss so that the directivity and HPBW values are lower than on the PA radar. Whereas the directivity and HPBW greatly influence the determination of MPSLL and the ability to minimize interference.

To overcome this weakness, the Tx-Rx subarrays approach for MIMO radar (TRSM) is proposed which utilizes a combination of overlapping subarray simultaneously on Tx and Rx arrays. Each subarray in the Tx-Rx array is equal. The waveform variation produced by the combination of the number of subarrays in the Tx-Rx array compensates for the beam shape loss and directivity. The optimum combination of waveform simultaneously increases directional coherent gain, and waveform diversity gain, so it has an impact on improvements in the radar performance such as minimum MPSSL, high directivity, narrow HPBW, and high SINR. 
Assuming a radar system with colocated antennas has $K$ antenna on the Tx array and $L$ antenna on the Rx array as in Fig. 4 [25, modification of Fig. 1]. Spaces between Tx and Rx antenna elements $d_{K}$ and $d_{L}$, respectively. $\mathrm{Tx}$ and $\mathrm{Rx}$ arrays have been partitioned into $M$ and $N$ subarrays which overlap one another. The number of antenna elements in each subarray is $K-M+1$ for the Tx array and $L-N+1$ for the Rx array. Each subarray works as a PA, so that each one forms a beam that goes in a certain direction. A beamforming weight vector is designed so as to maximize the coherent processing gain and waveform diversity gain on the subarray on the Tx array and also the Rx array expressed by $M \times 1$ coherent transmit vector $\mathbf{c}(\theta)$ and the transmit diversity vector $\mathbf{d}(\theta)$ and also $N \times 1$ receive coherent vector $\mathbf{g}(\theta)$ and diversity transmit vector $\mathbf{h}(\theta)$ to produce the $M N \times 1$ of T-R steering vector expressed by

$$
\mathbf{u}_{\mathrm{TRSM}}(\theta)=(\mathbf{c}(\theta) \circ \mathbf{d}(\theta)) \otimes(\mathbf{g}(\theta) \circ \mathbf{h}(\theta))
$$

with

$$
\begin{aligned}
\mathbf{g}(\theta) & =\left[\begin{array}{llll}
\mathbf{v}_{1}^{H} \mathbf{b}_{1}(\theta) & \mathbf{v}_{2}^{H} \mathbf{b}_{2}(\theta) & \cdots & \mathbf{v}_{N}^{H} \mathbf{b}_{N}(\theta)
\end{array}\right]^{T} \\
\mathbf{h}(\theta) & =\left[\begin{array}{llll}
e^{-j 2 \pi f \tau_{1}(\theta)} & e^{-j 2 \pi f \tau_{2}(\theta)} & \cdots & e^{-j 2 \pi f \tau_{N}(\theta)}
\end{array}\right]^{T}
\end{aligned}
$$

where $\mathbf{v}_{n}$ is the $L \times 1$ unit-norm complex weight vector for the $n$-th subarray in the Rx array with a definition similar to $\mathbf{w}_{m}, \mathbf{g}(\theta)$ is the $L \times 1$ receive coherent vector, and $\mathbf{h}(\theta)$ is the $L \times 1$ receive vector diversity.

In general the performance of the TRSM radar is expressed in Tx-Rx gain and SINR output [24 with alignment]. For the Tx-Rx gain of the TRSM radar is formulated with

$$
G_{\mathrm{TRSM}}(\theta)=(K / M)\left|\mathbf{u}_{\mathrm{TRSM}}^{H}(\theta) \mathbf{u}_{\mathrm{TRSM}}(\theta)\right|^{2}(7)
$$

whereas to determine the SINR output expressed by

$$
\operatorname{SINR}_{\mathrm{TRSM}}=\frac{(K / M) \sigma_{l}^{2}\left|\mathbf{w}_{\mathrm{r}}^{H} \mathbf{u}_{\mathrm{TRSM}}(\theta)\right|^{2}}{\mathbf{w}_{\mathrm{r}}^{H} \mathbf{R}_{i+n} \mathbf{w}_{r}}
$$

where $\mathbf{w}_{r}=\mathbf{u}_{\text {TRSM }}(\theta)$ and $\mathbf{R}_{i+n}$ is the matrix of covariance interference plus noise.

\section{Results and discussion}

In this simulation, it is assumed that the number of transmitter and receiver antennas is the same ( $K=$ $L$ ) with the number of subarrays in Tx and $\mathrm{Rx}$ arrays $1<M<K$ and $1<N<L$, respectively. The distance between the antenna elements in the transmitterreceiver is half wavelength. Target angle $\theta_{t}=0^{\circ}$. Noise is Gaussian with zero mean spatially and has the same variance on each antenna element.

For beampattern on the TRSM radar with $K=L=$ 30 and $M_{\mathrm{o}}=N_{\mathrm{o}}=15$, starting from transmit, waveform diversity, and transmit-receive shown in Fig. 5 (a). It appears that when null is at a certain angle of the beampattern transmit and is related to sidelobe at the beampattern waveform diversity or when it is null at a certain angle of the beampattern waveform diversity and is related to the sidelobe at the beampattern transmit it results in null at the T-R beampattern. So that by setting the null on the transmit or waveform diversity beampattern in such a way as to set the null on the T-R beampattern which is useful to minimize interference at certain locations.

In Fig. 5 (b), all the PMIMO radar models using only the subarray in the Tx array have the beamwidth of mainbeam tending to be wider than beamwidth of the PA radar. Although all of these radar models have MPSLL is much lower than the PA radar. Unlike the case with the TRSM radar which have a beamwith much narrower than all the PMIMO radar models, especially the PA radar. Likewise, the TRSM radar has the lowest MPSLL compared to all types of radars. So it is clear that the effect of applying subarray on Rx array to compensate for the weakness of the beam shape loss from transmit directional coherent gain and transmit diversity gain. In summary, the use of subarray methods on Tx and Rx arrays simultaneously produces the beamwidth of mainbeam with the lowest MPSLL without sacrificing its directivity. Also seen in Fig. 5 (c), if $K$ $=L$ on the TRSM radar increses, the narrower beamwidth at the mainbeam determines the directivity and HPBW. Another impact is the reduction in MPSLL compared to the PMIMO radar and their variants.

In Fig. 6 (a) shows the effect of increasing the target SNR with fixed INR $=30 \mathrm{~dB}$, strong interference condition, on the SINR output for all types of radars. It appears that the SINR output between the PA and the MIMO radar is the equal. This is in line with the results reported by [15]. This happens since both types of radar have similar PSLL levels on the T-R beampattern. Therefore the ability to minimize interference between the radars is also the alike. On the other hand, this radar appears to 


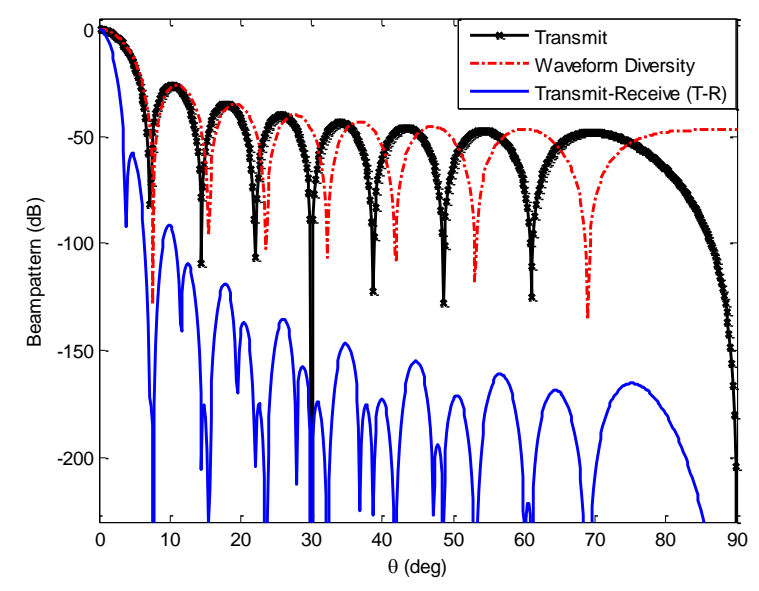

(a)

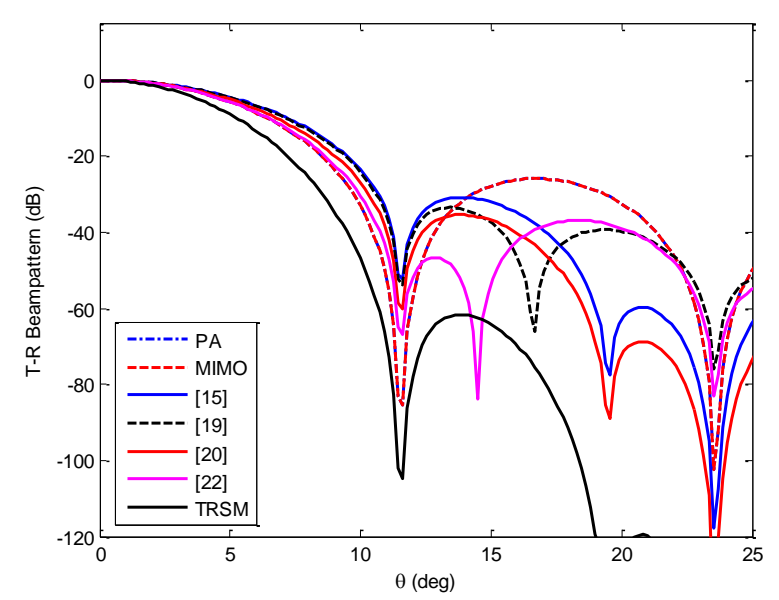

(b)

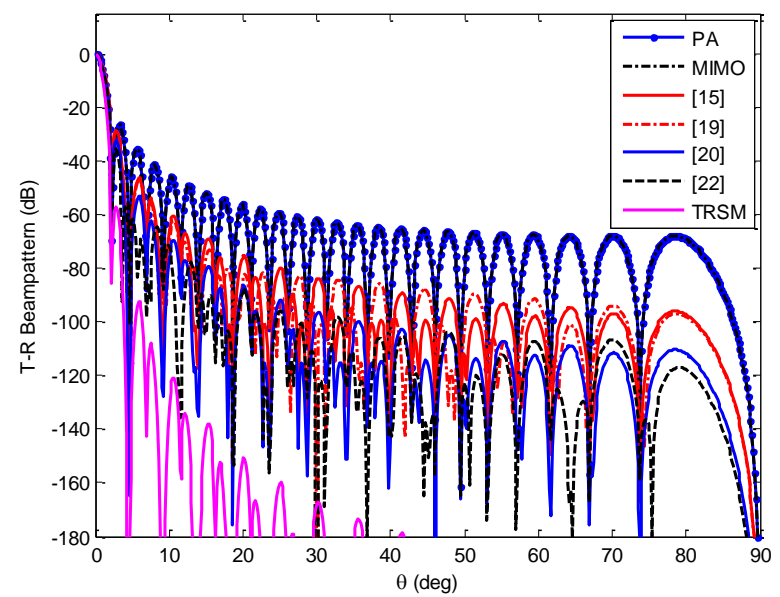

(c)

Figure. 5 Bempattern of the TRSM radar for: (a) transmit, waveform diversity, and T-R beampattern with $K=L=30$, (b) T-R beampattern for all radars with $K=L=10$, and (c) T-R beampattern for all radars for $K=L=50$

have higher SINR output compared to other types of the PMIMO radar. In comparison with study by [22], the SINR output of the TRSM radar is higher by about $9.34 \mathrm{~dB}$ than the SINR output of the OPPM radar.

Fig. 6 (b) shows the output SINR vs. SNR of all types of radars with a fixed value of $\mathrm{INR}=-30 \mathrm{~dB}$, a weak interference condition, i.e. the dominant noise power. It appears that the SINR output of the PA radar is ten times greater than the SINR output of the MIMO radar as stated by [15]. This happens because the PA radar provides directional coherent gain. The SINR output of the OPPM radar is much higher than all types of radars because the MPSLL it produces is lower than the MPSLL type of PMIMO radars. For the SINR output of the TRSM radar below the SINR output of the OPPM radar, this is due to a compromise of the transmit coherent gain and transmit diversity gain with receive coherent gain and receive diversity gain. In comparison to results of the OPPM radar [22], the SINR output of the radar is higher at $14.26 \mathrm{~dB}$ than the SINR output of the TRSM radar. In summary, for weak interference conditions, the SINR output performance of the OPPM radar is better than all types of radars.

Fig. 6 (c) shows the SINR output versus the INR for all types of radars with varying INR values (INR $=$ SNR). When the SNR is low, SINR output of the PA radar is higher than SINR output of the MIMO radar. Furthermore, when the SNR value increases, the difference in SINR output from the PA and the MIMO radars tends to decrease which eventually leads to the same value [15]. For the OPPM radar it has a higher SINR output when SNR is low i.e. SNR $=\mathrm{INR}=10 \mathrm{~dB}$ compared to SINR output of the TRSM radar. However, after SNR $=\mathrm{INR}=30 \mathrm{~dB}$, the SINR output of the TRSM radar is greater than $10.58 \mathrm{~dB}$ compared to SINR output of the OPPM radar. This shows that the SINR output performance of the TRSM radar becomes maximum suppressing interference when the interference power condition is greater than $30 \mathrm{~dB}$ and vice versa if the interference 


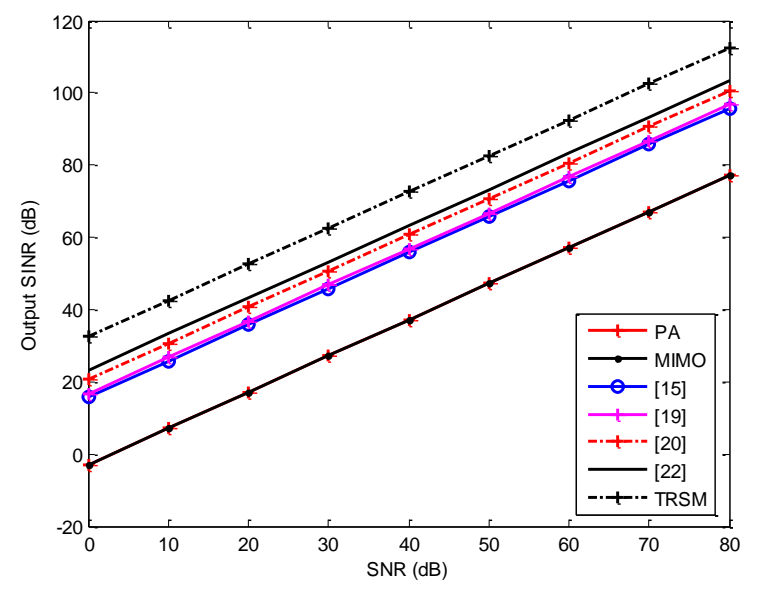

(a)

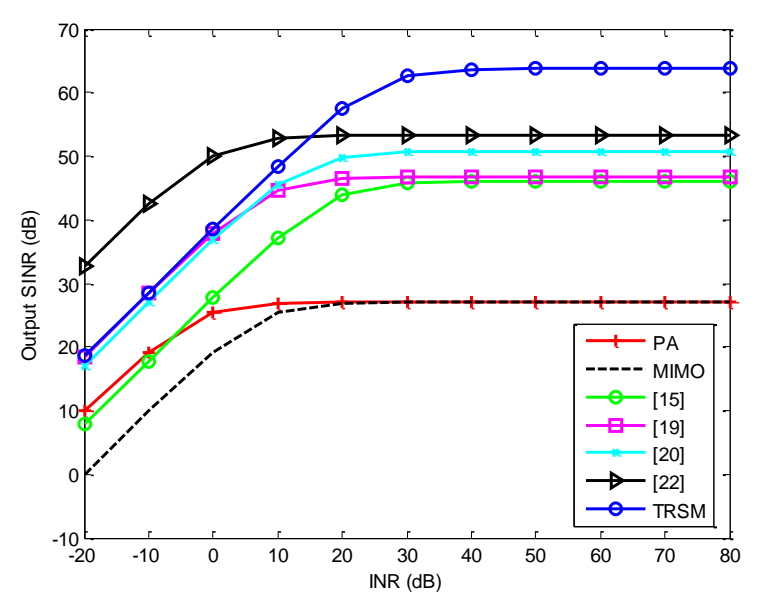

(c)

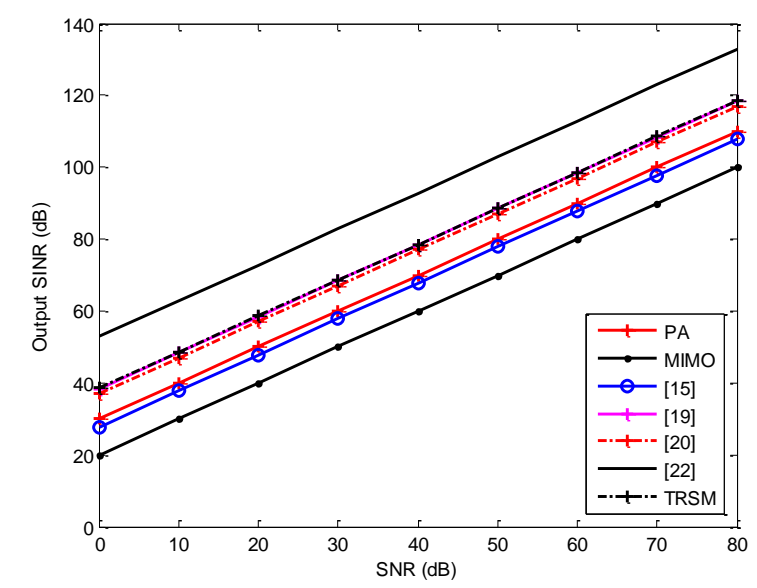

(b)

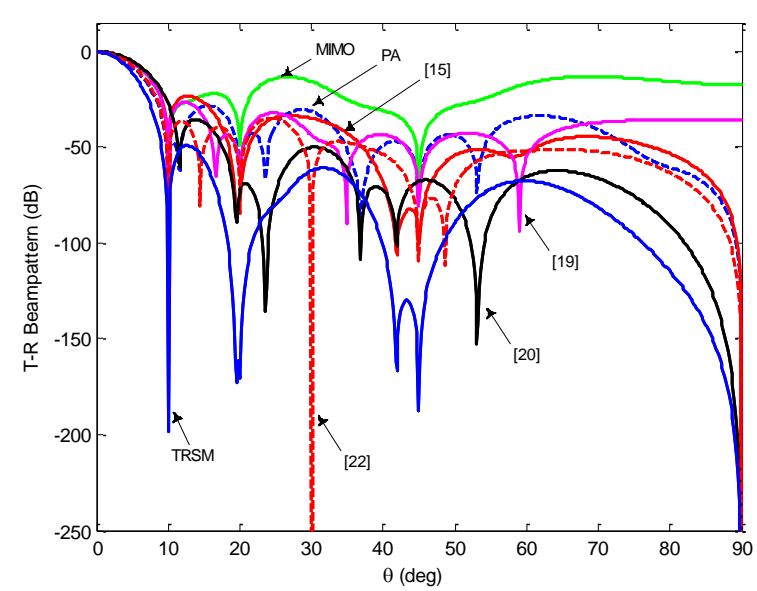

(d)

Figure. 6 Performance of all radars for: (a) Output SINR vs. SNR with fixed INR $=30 \mathrm{~dB}$, (b) Output SINR vs. SNR with fixed fixed INR $=-30 \mathrm{~dB}$, (c) output SINR versus INR $=$ SNR, and (d) T-R beampattern using MVDR beamformer

power condition is lower than $10 \mathrm{~dB}$ then the ability to minimize the interference is low. Also seen from Fig. 6 (c) that for the optimum number of subarrays $M=K / 2-1$ [19] it turns out that the SINR output values are almost the same between the PMIMO radar reported by [15] after SNR greater than $40 \mathrm{~dB}$.

To complete the discussion on the performance of PMIMO radar models, especially in dealing with interference, a T-R beampattern of all types of radar is presented using the MVDR (minimum variance distortionless response) beamformer as reported by [15], [20], and [21]. In Fig. 6 (d) shows the T-R beampattern of all types of radars with a target location of $0^{\circ}$ and some interference locations i.e. $10^{\circ}$, $20^{\circ}$, and $45^{\circ}$. The target power is $0 \mathrm{~dB}$ and interference power is $30 \mathrm{~dB}$. Appear at the locations of interference are all types of radars provide a nulling effect to suppress the smallest possible effect. The performance of the TRSM radar shows the superiority of all types of radars with the lowest MPSLL. This means that the performance of the TRSM radar is very robust against the presence of interference and at the same time also utilizes the advantages of Tx-Rx waveform diversity gain.

In Figs. 7 (a)-(c) show a comparison between all types of radar in terms of MPSLL, directivity, and HPBW for various $K$. It appears that the overall performance of the TRSM radar compared to the PMIMO radar in [15], the PMIMO $\operatorname{radar}(M=K / 2-$ 1) in [19], the HPMR-US radar in [20], the OPPM radar in [22] has MPSLL, directivity, and HPBW much better than other types of radars. The average MPSLL value on the TRSM radar is twice the MPSLL value on the PMIMO radar because the TRSM radar utilizes directional coherent and waveform diversity gain simultaneously in $\mathrm{Tx}$ array (such as the PMIMO radar) and also in Rx array. In general, the number of elements, $K=L$, appears to increase, so the MPSLL value tends to decrease for all types of radars. This tendency gives meaning to the demands of high MPSLL levels, so the number of antenna elements in the Tx-Rx array should not be large. Another result from Figs. 7 (a)-(c) which are 


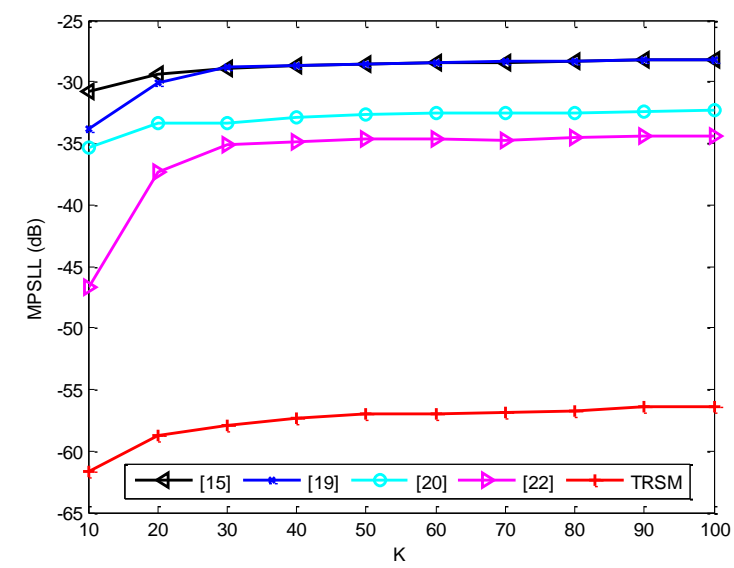

(a)

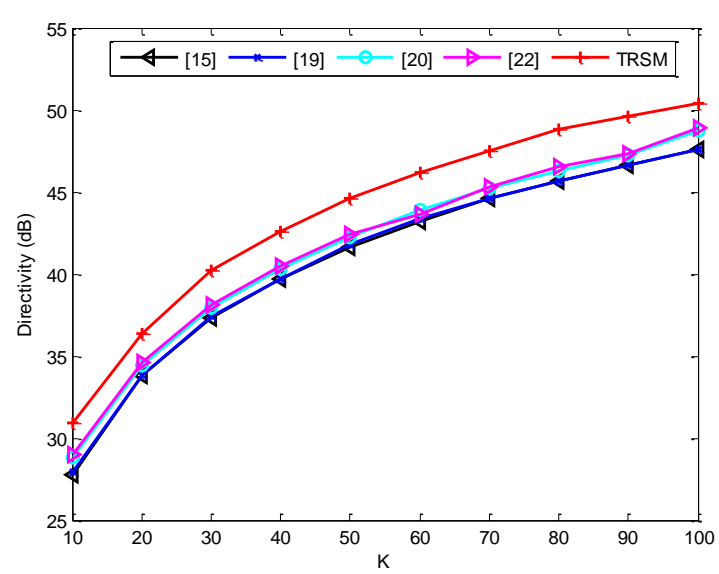

(b)

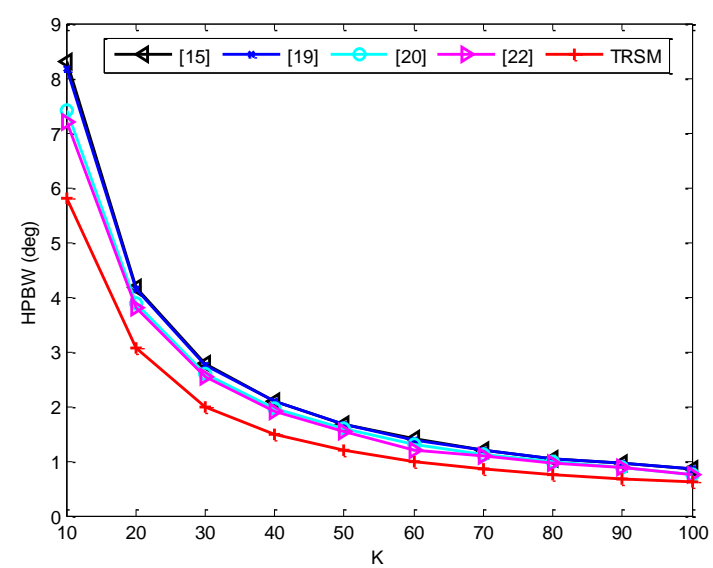

(c)

Figure. 7 Performance of all radars vs. number of total antennas $(K)$ for: (a) MPSLL, (b) directivity, and (c) HPBW

also important is that the TRSM radar has a low MPSLL without sacrificing directivity from the mainbeam. Thus with $K$ increasing, the value of MPSLL tends to decrease, the value of directivity tends to increase, and the value of HPBW tends to decrease.

\section{Conclusion}

In this paper a formula for $\mathrm{Tx}-\mathrm{Rx}$ subarrays at the MIMO radar (TRSM) has been formulated, including performance parameters, especially $\mathrm{Tx}-\mathrm{Rx}$ gain, SINR, MPSLL, directivity, and HPBW. Determination of the optimum $\mathrm{Tx}$ subarray (M) which affects the MPSLL, directivity, and HPBW for various $\mathrm{K}$ has also been presented and evaluated. The use of subarray methods on Tx and Rx arrays i.e. M and $\mathrm{N}$ simultaneously on the radar generates beamwidth from mainbeam with the lowest MPSLL without compromising its directivity so that a high SINR output is produced compared to other types of radars. In general there was an increase in the average performance of MPSLL, directivity, and HPBW vs. the number of subarrays for the radar relative to the OPPM radar is $21.5 \mathrm{~dB}, 2 \mathrm{~dB}$, and $0.45 \mathrm{deg}$. In the future, the use of Tx-Rx subarrays builds the TRSM radar more flexible and programmable to support the detection of multiple targets on array systems with multiple antennas. When implemented on a programmable platform such as software defined radio (SDR), etc. the flexibility of a radar system is easily realized.

\section{Conflicts of Interest}

I declare that in the preparation of this paper there is no conflict of interests in both circumstances and personal interests that can affect the representation or interpretation of all matters relating to the results of research. The author also declared no potential conflicts of interest with respect to the research, authorship, and/or publication of this article.

\section{References}

[1] M. I. Skolnik, Introduction to Radar Systems, $3^{\text {rd }}$ ed, McGraw-Hill, New York, NY, USA, 2001.

[2] M. S. Davis and A. D. Lanterman, "Coherent MIMO Radar: The Phased Array and Orthogonal Waveforms", IEEE Aerospace Electronic and Systems Magazine, Vol. 29, No. 8, pp. 76-91, 2014.

[3] A. Haimovich, R. Blum, and L. Cimini, "MIMO Radar with Widely Separated Antennas", IEEE Signal Processing Magazine, Vol. 25, No. 1, pp. 116-129, 2008.

[4] D. R. Fuhrmann and G. S. Antonio, "Transmit Beamforming for MIMO Radar Systems using Signal Cross-Correlation", IEEE Transactions on Aerospace and Electronic Systems, Vol. 44, No. 1, pp. 171-186, 2008.

[5] H. Chahrour, S. Rajan, R. Dansereau, and B. Balaji, "Hybrid Spread Spectrum Orthogonal 
Waveforms for MIMO Radar", In: Proc. of Radar Conf., Oklahoma City, OK, USA, pp. 1010-1014, 2018.

[6] S. Parnapalli and B. L. Malleswari, "Performance of MIMO MC-CDMA for STBC Communication System Using OKHA Based Optimal Channel Estimation", International Journal of Intelligent Engineering and Systems, Vol. 10, No. 4, pp. 1-10, 2017.

[7] S. Khaleelahmed and N. Venkateswararao, "Priority Based Scheduling for Energy Efficient Power Allocation in MIMO-NOMA System with Multiple Users", International Journal of Intelligent Engineering and Systems, Vol. 12, No. 4, pp. 348-357, 2019.

[8] J. Li, P. Stoica, X. Luzhou, and W. Roberts, "On Parameter Identifiability of MIMO Radar", IEEE Signal Processing Letters, Vol. 14, No. 12, pp. 968 - 971, 2007.

[9] L. Wang, Y. Zhang, Q. Liao, and J. Tang, "Robust Waveform Design for Multi-Target Detection in Cognitive MIMO Radar", In: Proc. of Radar Conf., Oklahoma City, OK, USA, pp. 116-120, 2018.

[10] E. Brookner, "MIMO Radar for Automobiles", In: Proc. of International Symposium on Phased Array System and Technology, Waltham, MA, USA, pp. 1-5, 2019.

[11] J. Misiurewicz, Ł. Ma'slikowski, A. Gromek, and A. Kurowska, "MIMO Techniques for Space Surveillance Radar", In: Proc. of Signal Processing Symposium, Krakow, Poland, pp. 199-202, 2019.

[12] K. Kulpa and L. Maslikowski, "Detection Range Limitation in MIMO and SISO Noise Radar", In: Proc. of International Microwave and Radar Conf., Poznan, Poland, pp. 471-472, 2018.

[13] Y. C. Lin, T. S. Lee, Y. H. Pan, and K. H. Lin, "Low-Complexity High-Resolution Parameter Estimation for Automotive MIMO Radars", IEEE Access, Vol. 8, pp. 16127-16138, 2020.

[14] K. H. Kim, H. Kim, D. Y. Kim, S. K. Kim, S. H. Chun, S. J. Park, S. M. Jang, M. K. Chong, and H. S. Jin, "Development of Planar Active Phased Array Antenna for Detecting and Tracking Radar", In: Proc. of Radar Conf., Oklahoma City, OK, USA, pp. 100-103, 2018.

[15] A. Hassanien and S. A. Vorobyov, "PhasedMIMO Radar: A Tradeoff between PhasedArray and MIMO Radars", IEEE Transactions on Signal Processing, Vol. 58, No. 6, pp. 31373151, 2010.

[16] T. D. Backes, "Parameter Identifiability in Phased-Subarray MIMO Radar", In: Proc. of
Aerospace Conf., Big Sky, Montana, pp. 1-6, 2014.

[17] W. Q. Wang, "Phased-MIMO Radar with Frequency Diversity for Range-Dependent Beamforming", IEEE Sensors Journal, Vol. 13, No. 4, pp. 1320-1328, 2013.

[18] D. R. Fuhrmann, J. P. Browning, and M. Rangaswamy, "Signaling Strategies for the Hybrid MIMO Phased-Array Radar", IEEE Journal of Selected Topics in Signal Processing, Vol. 4, No. 1, pp. 66-78, 2010.

[19] N. E. D. Ismail, S. H. Mahmoud, A. S. Hafez, and T. Reda, "A New Phased MIMO Radar Partitioning Schemes", In: Proc. of Aerospace Conf., Big Sky, Montana, pp. 1-7, 2014.

[20] W. Khan, I. M. Qureshi, A. Basit, and M. Zubair, "Hybrid Phased MIMO Radar with Unequal Subarrays", IEEE Antennas and Wireless Propagation Letters, Vol. 14, pp. 1702-1705, 2015.

[21] S. Tahcfulloh and G. Hendrantoro, "PhasedMIMO Radar using Hadamard Coded Signal", In: Proc. of International Conf. On Radar, Antenna, Microwave, Electronics, and Telecommunications, Jakarta, Indonesia, pp. 1316, 2016.

[22] A. Alieldin, Y. Huang, and W. M. Saad, "Optimum Partitioning of a Phased-MIMO Radar Array Antenna", IEEE Antennas and Wireless Propagation Letters., Vol. 16, pp. 2287-2290, 2017.

[23] S. Tahcfulloh and G. Hendrantoro, "Phased MIMO Radar with Coherent Receive Arrays", In: Proc. of International Conf. on Signals and Systems, Sanur, Indonesia, pp. 72-76, 2017.

[24] S. Tahcfulloh and G. Hendrantoro, "Full Phased MIMO Radar with Colocated Antennas", International Journal on Communications Antenna and Propagation, Vol. 9, No. 2, pp. 144-154, 2019.

[25] S. Tahcfulloh and G. Hendrantoro, "FPMIMO: A General MIMO Structure with Overlapping Subarrays for Various Radar Applications", IEEE Access, Vol. 8, pp. 11248-11267, 2020. 